\title{
Experiencia de búsqueda en cibermedios: análisis comparativo de diarios nativos digitales
}

\author{
Carlos Lopezosa*, Mar Iglesias-García**, Cristina González-Díaz**, Lluís Codina* \\ *Universitat Pompeu Fabra \\ Correo-e: carlos.lopezosa@upf.edu | ORCID iD: https://orcid.org/0000-0001-8619-2194 \\ Correo-e: Iluis.codina@upf.edu | ORCID iD: https://orcid.org/0000-0001-7020-1631 \\ **Universidad de Alicante \\ Correo-e: mar.iglesias@gcloud.ua.es | ORCID iD: https://orcid.org/0000-0001-7926-5746 \\ Correo-e: cristina.gdiaz@ua.es | ORCID iD: https://orcid.org/0000-0001-6981-0499
}

Recibido: 14-04-2019; 2a versión: 04-06-2019; Aceptado: 05-06-2019.

Cómo citar este artículo/Citation: Lopezosa, C.; Iglesias-García, M.; González-Díaz, C.; Codina, L. (2020). Experiencia de búsqueda en cibermedios: análisis comparativo de diarios nativos digitales. Revista Española de Documentación Científica, 43 (1), e254. https://doi.org/10.3989/redc.2020.1.1677

Resumen: El objetivo principal de este estudio es el análisis de los componentes que proporcionan buscabilidad y visibilidad a los diarios nativos digitales en Internet, bajo el concepto unificador de la experiencia de búsqueda. La metodología empleada es el análisis comparativo del así llamado Search Experience Optimization (SXO) de un grupo de medios digitales. En concreto, se han analizado eldiario.es, publico.es, elconfidencial.com, elespanol.com, huffingtonpost. es, infolibre.es, libertaddigital.com y vozpopuli.com. Para llevar a cabo este análisis utilizamos el protocolo de estudio denominado PAXBCM. Gracias a este análisis hemos podido mapear e identificar diferentes grados de cumplimiento de la SXO por parte de los cibermedios analizados, siendo libertaddigital.com el que presenta mejores resultados globales. Todos los medios analizados presentan oportunidades de mejora en diversos aspectos, especialmente en la velocidad de carga, el uso de contenido multimedia, la accesibilidad y la buscabilidad.

Palabras clave: SEO; UX; Search Experience Optimization; posicionamiento en buscadores; periodismo digital; cibermedios.

\section{Searching experience in cibermedia: comparative analysis of digital journals}

Abstract: The aim of this study is the analysis of the components that provide searchability and visibility to digitalborn news media on the Internet, under the unifying concept of the search experience. The methodology used is a comparative analysis of the so-called Search Experience Optimization (SXO) applied to a group of digital news media. Specifically, eldiario.es, publico.es, elconfidencial.com, elespanol.com, huffingtonpost.es, infolibre.es, libertaddigital.com and vozpopuli.com have been analyzed. To carry out this analysis, we have used the study protocol called PAXBCM. Thanks to this analysis we have been able to confirm different degrees of compliance with SXO by the digital news media analyzed, being libertaddigital.com the one with the best overall results. However, all the cases studied present opportunities for improvement in various aspects such as loading speed, use of multimedia content, accessibility, and searchability.

Keywords: SEO; UX; Search Experience Optimization; Search Engine Optimization; digital journalism; digital news media.

Copyright: (c) 2020 CSIC. Este es un artículo de acceso abierto distribuido bajo los términos de la licencia de uso y distribución Creative Commons Reconocimiento 4.0 Internacional (CC BY 4.0). 


\section{INTRODUCCIÓN}

La visibilidad o posicionamiento web es uno de los principales retos que deben superar los medios de comunicación para atraer a los lectores que utilizan buscadores, tales como Google o Bing, para acceder a la información de actualidad (Giomelakis y Veglis, 2015a; 2015b; Charlton, 2016; GarcíaCarretero y otros, 2016).

Como han confirmado otros trabajos (Asser, 2012; Smyrnaios, 2015), existe una serie de procedimientos y técnicas agrupadas bajo la denominación tradicional de Search Engine Optimization (SEO) que ayuda a que el contenido de una página web ocupe mejores posiciones en los resultados de búsqueda (Lopezosa y otros, 2018). Sin embargo, el SEO tradicional está evolucionando hacia una nueva versión que amplía el foco de atención a aspectos que afectan principalmente a la interfaz de usuario, a la navegación interna y a la buscabilidad (entre otros) y que se denomina Optimización de la Experiencia de Búsqueda - Search Experience Optimization (en adelante SXO). Los autores de este trabajo creemos que el cambio de Search Engine (buscador) por Search Experience es muy importante porque insiste en mejorar aspectos del sistema que afectan a los usuarios y no solo a los buscadores.

Uno de los aspectos que valoramos es que el SXO permite una intersección de intereses entre la visibilidad en la Web (buscadores) y el acceso a la información (navegación y buscabilidad) en el interior de cada medio de comunicación. Mientras que la visibilidad incluye los componentes del SEO más tradicionales como pudieran ser contenido optimizado para motores de búsqueda, optimización de palabra clave, etc., el acceso a la información en el interior del propio sitio web aborda otros aspectos como la arquitectura de la información, las taxonomías, el buscador interno, etc.

Los principales trabajos anteriores sobre SXO (Cutts, 2012; Potter, 2012; Villanueva, 2013; Munroe, 2015; Arora, 2016; Kukoo, 2016; Templeman, 2016; Chasinov, 2017) indican la posibilidad de realizar estudios de caso y análisis comparativos, cosa que nosotros llevamos a los cibermedios, abriendo así el foco de los estudios del SEO al aplicarlo a los medios de comunicación digitales.

Para nosotros, una de las principales motivaciones es que, en el contexto digital, incluso el mejor periodismo puede resultar perjudicado si sus contenidos no llegan de forma eficaz a los lectores. Esto puede suceder si queda sepultado por contenidos del mismo tema, pero de inferior calidad que, sin embargo, pueden ocupar las primeras páginas de resultados de los buscadores y de las redes sociales.

\subsection{Objetivos}

Ante este contexto, el objetivo principal de este trabajo es analizar los componentes de la experiencia de búsqueda de sitios web de medios de comunicación digitales, y más concretamente de los diarios nativos digitales, puesto que es el grupo de factores que de forma más decisiva puede influir en la visibilidad de las noticias. Para ello, se analizan los siguientes medios: eldiario.es, publico.es, elconfidencial.com, elespanol.com, huffingtonpost. es, infolibre.es, libertaddigital.com y vozpopuli. com. A partir de este objetivo principal, planteamos los siguientes tres objetivos concretos:

1. Identificar los componentes del SXO, tanto en la versión móvil como ordenador de escritorio, en los principales diarios nativos digitales de España y determinar la eficacia de su aplicación.

2. Desarrollar y llevar a cabo un análisis comparativo con capacidad para proporcionar un ranking que identifique los medios que llevan a cabo mejores prácticas en los diferentes parámetros del SXO.

3. Proponer recomendaciones de SXO susceptibles de aplicarse a medios de comunicación digitales.

\subsection{Pregunta de investigación}

La pregunta de investigación que nos hemos formulado, atendiendo a los objetivos señalados, es la siguiente:

- ¿Cuáles son los componentes más significativos del SXO, cuál es el grado de observación e implementación de estos componentes que presentan los diarios nativos digitales españoles y cuál es la posición relativa de cumplimiento entre ellos?

El motivo que nos lleva a plantear esta pregunta es el convencimiento, apoyado por los múltiples análisis sobre el uso de buscadores por parte del público (Morato y otros, 2013), de que tanto la visibilidad como el acceso a la información en el interior de cada sitio forman parte del compromiso de calidad que los medios de comunicación deben tener con sus lectores.

Como observación final a propósito de la oportunidad de esta clase de análisis, entendemos que el encaje de los cibermedios en el ecosistema digital debe considerar todas las propiedades del canal o del soporte. Tal como la radio, por poner un ejemplo, debe tener en cuenta las características del canal de audio, los medios digitales deben tener en cuenta aspectos como la visibilidad y el acceso a la información, y de aquí el interés intrínseco que tienen las investigaciones sobre el SEO en general, y sobre el SXO en particular, aplicado a cibermedios. 


\section{MARCO TEÓRICO}

Una parte muy importante de los usuarios de la Web utilizan buscadores como Google, Bing o Yahoo! como parte de su dieta digital (Sampedro, 2018). Para ello, realizan búsquedas desde estas páginas y consumen los resultados que se ubican en primeras posiciones, obviando aquellos que no ocupan estos lugares preferentes.

Ante esta circunstancia, los cibermedios intentan que sus noticias aparezcan en estas posiciones privilegiadas para obtener un mayor número de lectores y por tanto mejores oportunidades para su sostenibilidad. Sin embargo, para aumentar las posibilidades de conseguirlo además de los mejores contenidos posibles, necesitan aplicar una serie de estrategias SEO (IglesiasGarcía y Codina, 2016).

Estas técnicas de SEO han evolucionado hacia una nueva forma de entender la optimización en los motores de búsqueda. La principal razón se debe a que los buscadores son cada vez más sofisticados y están aprendiendo a entender el comportamiento de los usuarios (Hassan y otros, 2004; Van Dyke, 2012; Arora, 2016; Jiménez y otros, 2018), o lo que es lo mismo, consiguen adaptarse cada vez mejor a estos usuarios (Themistoklis y otros, 2015; Liu, 2017). Esta evolución del SEO es identificada como SXO, esto es, un conjunto de factores que tienen como objetivo optimizar la experiencia de búsqueda integral de los usuarios.

Por tanto, son factores que intervienen no solamente cuando el usuario envía búsquedas a Google, sino también cuando navega por el contenido de un sitio web (Villanueva, 2013; Lemos y Yoseph, 2017; Park, 2018) con el fin de acceder a las informaciones publicadas en el mismo. A su vez, todo esto implica que cuanto más se optimice esta experiencia, mayores posibilidades tienen los sitios web de aparecer en los primeros puestos de los buscadores, ya que estos han desarrollado medios para incorporar las señales de los usuarios a sus criterios de ordenación de resultados.

Como sea, lo cierto es que, aunque el SXO y su definición proviene de fuentes muy diversas (Cutts, 2012; Potter, 2012; Villanueva, 2013; Munroe, 2015; Arora, 2016; Chasinov, 2017), hay una notable confluencia en los aspectos principales que forman el mismo, lo que nos permite desarrollar y aplicar herramientas de análisis como la que propondremos.

En concreto, el primer experto en referirse al SXO fue el director del departamento de lucha contra el spam de Google, el ingeniero Matt Cutts en el año 2012. En concreto, declaró que, ante el avance interpretativo de los algoritmos de Google en el tratamiento de las búsquedas de los usuarios, los expertos en optimización en buscadores debían plantearse nuevas formas de trabajo más enfocadas en el usuario y menos en el buscador, naciendo así, probablemente por primera vez, el concepto SXO (Cutts, 2012).

Un aspecto importante a considerar aquí es que no solo Google y otros buscadores han marcado la evolución del SEO al SXO, sino que también fueron esenciales para dicha evolución otras importantes influencias, como las redes sociales y el consumo de Internet a través de nuevos dispositivos como los móviles y tabletas (Manish y Roohi, 2013).

En síntesis, el SXO es el conjunto de factores que pueden mejorar aspectos relacionados con la interacción de los usuarios con un sitio (Potter, 2012; Munroe, 2015; Pérez-Montoro y Codina, 2016; Gandhi, 2017; Codina, 2018), y que por su naturaleza, trasladan señales que los buscadores identifican y les permiten privilegiar a los sitios que están mejor optimizados en este sentido.

\section{METODOLOGÍA}

Para realizar esta investigación hemos llevado a cabo un estudio de caso múltiple (Yin, 2014; Coller, 2000; Eisenhardt, 1989) sobre los principales diarios nativos digitales españoles. Para su selección, hemos tomado como indicador su relevancia en el ranking de Comscore (empresa auditora de medios de comunicación digital).

Por su parte, para analizar estos diarios nativos digitales hemos utilizado el Protocolo de Análisis de la eXperiencia de Búsqueda en Cibermedios, o PAXBCM (Lopezosa y Codina, 2020), diseñado para permitir el análisis comparativo de la optimización de la experiencia de búsqueda en cibermedios. PAXBCM utiliza, por un lado, tanto el Sistema Articulado de Análisis de Cibermedios O SAAC (Codina y otros, 2014) como el Sistema de la Evaluación de Recursos Digitales en Línea (Codina, 2000). Asimismo, para su confección hemos consultado también la propuesta del Sistema de análisis de hemerotecas de prensa digital (Guallar y otros, 2012), así como el sistema de acceso y consulta en los diarios digitales españoles (Nuño, 2014) y el protocolo de análisis de periodismo en bases de datos y buscabilidad de la información (Linares y otros, 2016). De este modo, cada uno de los parámetros utilizados en el análisis y la puntuación que otorgan sus indicadores siguen las pautas principales marcadas por las metodologías señaladas, especialmente el SAAC. 


\subsection{Objeto de estudio}

Presentamos a continuación, mediante fichas sistematizadas, los medios que han sido seleccionados por tratarse de los diarios nativos digitales (Tabla I), que son los que ocupan las mejores posiciones en el ranking en base a su número de lectores.

Para estimar este ranking, hemos utilizado los datos proporcionados por Comscore, tras descartar el uso de los informes de la Oficina de Justificación de la Difusión (OJD). La razón es que no todos los diarios nativos digitales se encuentran registrados en OJD. De hecho, en el caso que nos ocupa solo cuatro de los ocho nativos digitales estaban presentes en OJD Interactiva. Por tanto, para poder utilizar estimaciones homogéneas hemos optado por Comscore. Cabe señalar que, según nuestra estimación, el grupo finalmente seleccionado no hubiera variado en caso de que todos los nativos digitales hubieran estado registrados en OJD.
Por último, debemos señalar que, para acceder a los datos de Comscore, hemos utilizado la publicación de los informes de OK Diario (2016 y 2018) y Prensa PR (2018) en donde se recogen la posición en el ranking de los principales cibermedios en base a su número de lectores.

\subsection{Protocolo de análisis}

Seguidamente, presentamos los parámetros e indicadores del protocolo PAXBCM (Tabla II) que se utilizarán para analizar los medios de comunicación seleccionados. El análisis se llevó a cabo entre el 9 de febrero y el 10 de marzo de 2019.

En PAXBCM, cada parámetro se articula en una serie de indicadores, que son los que permiten operacionalizar los análisis. En total, PAXBCM contempla 26 indicadores. La lista completa de los mismos, su descripción, la pregunta de chequeo y su puntuación teórica se presentan en la tabla III.

Tabla I. Diarios nativos digitales analizados. Vinculación empresarial y posición por número de lectores en el ranking Comscore

\begin{tabular}{|c|c|c|c|}
\hline ID & Nombre & Vinculado con & Ranking Comscore \\
\hline M1 & eldiario.es & Associated Whistleblowing Press & 9 (datos de 2018) \\
\hline M2 & publico.es & Display Connectors & 13 (datos de 2018) \\
\hline M3 & elconfidencial.com & Titania Compañía Editorial, S. L. & 4 (datos de 2018) \\
\hline M4 & elespanol.com & Nohacefaltapapel & 8 (datos de 2018) \\
\hline M5 & huffingtonpost.es & Arianna Huffington, Kenneth Lerer, PRISA & 12 (datos de 2018) \\
\hline M6 & infolibre.es & Mediapart, editorial Edhasa & 27 (datos de 2016) \\
\hline M7 & libertaddigital.com & Libertad Digital S.A. & 11 (datos de 2018) \\
\hline M8 & vozpopuli.com & Vozpópuli Digital, S. L. & 37 (datos de 2016) \\
\hline
\end{tabular}

Fuente: Datos de Comscore.

Tabla II. Parámetros del protocolo PAXBCM para el análisis de los diarios nativos digitales

\begin{tabular}{|c|l|}
\hline \multicolumn{2}{|c|}{ Descripción de los parámetros del protocolo de análisis de SXO en cibermedios } \\
\hline Parámetro & \multicolumn{1}{c|}{ Definición } \\
\hline $\begin{array}{c}\text { Arquitectura de la } \\
\text { información }\end{array}$ & $\begin{array}{l}\text { La arquitectura de la información es la buena disposición y estructuración de la información de un } \\
\text { sitio web. }\end{array}$ \\
\hline $\begin{array}{c}\text { SEO de } \\
\text { contenidos }\end{array}$ & $\begin{array}{l}\text { El SEO de contenidos es el conjunto de técnicas aplicadas al contenido de una web que ayudan al mis- } \\
\text { mo tiempo a mejorar su calidad y su posicionamiento en los buscadores como Google, Bing, etcétera. }\end{array}$ \\
\hline SEO técnico & $\begin{array}{l}\text { El SEO técnico es un campo dentro de la optimización del posicionamiento en buscadores } \\
\text { centrada en la mejora de la parte más tecnología de una web como pudiera ser la optimización } \\
\text { del código fuente, la configuración del servidor, etc. }\end{array}$ \\
\hline Metadatos & $\begin{array}{l}\text { Los Metadatos son etiquetas en HTML que describen información para que ésta sea entendida de } \\
\text { manera eficaz por los motores de búsqueda. }\end{array}$ \\
\hline $\begin{array}{c}\text { Señales de } \\
\text { usuario }\end{array}$ & $\begin{array}{l}\text { Las señales de usuarios son un conjunto de indicadores que identifican y miden el comportamiento } \\
\text { de los usuarios que navegan por una página web. Estos indicadores van desde el tiempo de } \\
\text { navegación de un usuario por una web, el número de páginas internas que visita, etcétera. }\end{array}$ \\
\hline Autoridad & $\begin{array}{l}\text { La Autoridad web es un indicador SEO que cuantifica el grado de popularidad de un sitio web en } \\
\text { base a las referencias recibidas en forma de enlaces desde otras páginas web. }\end{array}$ \\
\hline Buscabilidad & $\begin{array}{l}\text { La buscabilidad es el campo que se encarga de estudiar la eficacia y las técnicas necesarias para } \\
\text { que los usuarios encuentren información específica tanto dentro como fuera de una página web. }\end{array}$ \\
\hline
\end{tabular}

Fuente: Adaptado de Lopezosa y Codina (2019). 
Tabla III. Fichas descriptivas de los indicadores que componen cada uno de los parámetros de PAXBCM y su puntuación teórica

\section{PRESENTACIÓN DE LOS INDICADORES DEL MODELO DE ANÁLISIS DE SXO}

\section{Parámetro 1: Arquitectura de la información}

\begin{tabular}{|c|c|c|c|}
\hline ID & Indicador & Descripción & Puntuación \\
\hline 1.1 & $\begin{array}{l}\text { Navegación } \\
\text { estructural }\end{array}$ & $\begin{array}{l}\text { La navegación estructural permite comprender el contenido principal del sitio } \\
\text { y hacer el recorrido por las secciones principales del portal con un máximo } \\
\text { de tres clics. Debe tener un número limitado de componentes (alrededor de } \\
\text { 10) y debe ser constante: se mantiene sin cambios a lo largo del sitio. ¿El } \\
\text { sitio web cuenta con una navegación que responda a los criterios señalados } \\
\text { en la definición? }\end{array}$ & $0-1$ \\
\hline 1.2 & $\begin{array}{c}\text { Enlaces } \\
\text { semánticos }\end{array}$ & $\begin{array}{l}\text { Los enlaces semánticos se establecen en base a la similitud temática entre } \\
\text { contenidos de diferentes páginas. Suelen mostrarse como contenido relacio- } \\
\text { nado en algunas noticias mediante enlaces separados del texto principal, pero } \\
\text { asociados al mismo. ¿La web, tanto en su versión móvil como en su versión } \\
\text { de escritorio, cuenta con una navegación que responda a los criterios seña- } \\
\text { lados en la definición? }\end{array}$ & $0-1$ \\
\hline 1.3 & Taxonomía & $\begin{array}{l}\text { Una taxonomía es un esquema de organización de la información que permi- } \\
\text { te mantener unidas las noticias sobre el mismo tema. Una taxonomía debe } \\
\text { incluir al menos categorías y subcategorías. Puede incluir etiquetas. Las cate- } \\
\text { gorías y subcategorías agrupan generalmente las secciones de la web, y las } \\
\text { etiquetas agrupan las ideas que se han abordado en el contenido publicado } \\
\text { en el sitio web. ¿La web, tanto en su versión móvil como en su versión de } \\
\text { escritorio, presenta una navegación basada en el uso de categorías y, opcio- } \\
\text { nalmente, etiquetas? }\end{array}$ & $0-1$ \\
\hline 1.4 & Enlazado interno & $\begin{array}{l}\text { El enlazado interno es el conjunto de enlaces que se crean entre noticias re- } \\
\text { lacionadas de una misma web con el objetivo de facilitar una navegación am- } \\
\text { pliada al usuario. Se distingue de la navegación semántica porque el enlazado } \\
\text { interno está incrustado en el cuerpo de la noticia. Por tanto, son enlaces que } \\
\text { deben ser altamente relevantes ¿Las noticias publicadas en la web analizada } \\
\text { cuentan con enlaces internos? }\end{array}$ & $0-1$ \\
\hline \multicolumn{4}{|c|}{ Parámetro 2: SEO de contenidos } \\
\hline ID & Indicador & Descripción & Puntuación \\
\hline 2.1 & Extensión & $\begin{array}{l}\text { Noticias con } 400 \text { o más palabras. Los contenidos de extensión media-larga } \\
\text { indican calidad porque implican mayor profundización en el tratamiento de los } \\
\text { temas. ¿El sitio cuenta con contenidos de } 400 \text { o más palabras? }\end{array}$ & $0-1$ \\
\hline 2.2 & Palabras clave & $\begin{array}{l}\text { Las palabras clave son términos que utilizan los usuarios cuando buscan in- } \\
\text { formación en Internet. Se componen por una o más palabras y su existencia } \\
\text { en las páginas de las noticias permiten a los buscadores entender el conteni- } \\
\text { do y elegirlo para incluirlo en sus resultados cuando el usuario hace consultas } \\
\text { a temas relacionados. ¿La noticia seleccionada cuenta con palabras clave } \\
\text { derivadas que enriquecen el titular y/o el tema de la noticia? }\end{array}$ & $0-1$ \\
\hline 2.3 & $\begin{array}{l}\text { Contenido } \\
\text { audiovisual }\end{array}$ & $\begin{array}{l}\text { Dadas las características de Internet, los contenidos de calidad incluyen ma- } \\
\text { teriales multimedia. El material audiovisual puede formar parte del contenido } \\
\text { de una noticia. Con ello no solo se mejora el diseño haciéndolo más atractivo, } \\
\text { sino que además permite enriquecer y complementar el contenido de la web. } \\
\text { ¿El sitio cuenta con contenidos audiovisuales que complementa el contenido } \\
\text { de la noticia? }\end{array}$ & $0-1$ \\
\hline 2.4 & Mobile First & $\begin{array}{l}\text { Mobile First es una iniciativa que pretende llevar a cabo diseños web pen- } \\
\text { sados primero en móvil y después adaptados a la versión escritorio. Actual- } \\
\text { mente, se trabaja al revés, primero pensando en la versión para ordenador } \\
\text { y después adaptándola a la versión móvil. ¿La web analizada participa de la } \\
\text { filosofía Mobile First? }\end{array}$ & $0-1$ \\
\hline 2.5 & Accesibilidad & $\begin{array}{l}\text { La accesibilidad web consiste en crear una web sin barreras, esto es, que per- } \\
\text { mite su acceso a personas con diferentes discapacidades. ¿La web analizada } \\
\text { cuenta con una buena accesibilidad web? }\end{array}$ & $0-1$ \\
\hline
\end{tabular}




\begin{tabular}{|c|c|c|c|}
\hline \multicolumn{4}{|c|}{ Parámetro 3: SEO técnico } \\
\hline ID & Indicador & Descripción & Puntuación \\
\hline 3.1 & $\begin{array}{l}\text { Página de error } \\
\quad 404\end{array}$ & $\begin{array}{l}\text { El error } 404 \text { es un código de estado HTTP que se envía desde el servidor de } \\
\text { una web al navegador, y le dice al usuario que la url introducida no existe o } \\
\text { es errónea. Algunas webs crean una página personalizada cuando se da este } \\
\text { error para ayudar al usuario a encontrar el contenido que deseaba. Cuando } \\
\text { se introduce un enlace erróneo o no existente de la web caso de estudio ¿nos } \\
\text { lleva a una página personalizada de error } 404 \text { o no? }\end{array}$ & $0-1$ \\
\hline 3.2 & $\begin{array}{l}\text { Protocolo robots. } \\
\text { txt }\end{array}$ & $\begin{array}{l}\text { Robots.txt es un archivo de texto que se sube a la web y se utiliza para im- } \\
\text { pedir que los motores de búsqueda rastreen e indexen contenido que no que- } \\
\text { remos ni que sea indexado ni que sea rastreado. ¿La web analizada cuenta } \\
\text { con el archivo robots.txt? }\end{array}$ & 0.1 \\
\hline 3.3 & Mapa del sitio $\mathrm{xml}$ & $\begin{array}{l}\text { El mapa del sitio web o sitemap xml es un archivo que contienen un listado } \\
\text { de todas las páginas de un sitio web. Este listado ayuda a los motores de bús- } \\
\text { queda a localizar de manera sencilla y con menos recursos cada uno de los } \\
\text { enlaces que componen el sitio web, facilitando la indexación en sus índices. } \\
\text { ¿La web analizada cuenta con el archivo sitemap xml? }\end{array}$ & $0-1$ \\
\hline 3.4 & $\begin{array}{l}\text { Velocidad de } \\
\text { carga }\end{array}$ & $\begin{array}{l}\text { La velocidad de carga de una web es el tiempo estimado que tarda un sitio } \\
\text { en cargarse. La velocidad depende principalmente del peso de la web, por lo } \\
\text { que cuanto menos pese más rápido cargará. ¿La velocidad de carga de la web } \\
\text { analizada está optimizada? }\end{array}$ & $0-0.5-1$ \\
\hline \multicolumn{4}{|c|}{ Parámetro 4: Metadatos } \\
\hline ID & Indicador & Descripción & Puntuación \\
\hline 4.1 & $\begin{array}{c}\text { Atributo ALT en la } \\
\text { imagen }\end{array}$ & $\begin{array}{l}\text { El atributo alt es un atributo que nos permite describir una imagen subida a } \\
\text { una web y tiene la función de explicarle a los motores de búsqueda en qué } \\
\text { consiste la imagen publicada. ¿Las imágenes de las noticias seleccionadas } \\
\text { de cada uno de los medios caso de estudio cuentan con su atributo alt im- } \\
\text { plementado? }\end{array}$ & $0-1$ \\
\hline 4.2 & $\begin{array}{l}\text { Marcado } \\
\text { semántico con } \\
\text { Schema.org }\end{array}$ & $\begin{array}{l}\text { Schema.org es una iniciativa desarrollada por Google, BING, y Yahoo, que } \\
\text { permite marcar semánticamente el contenido de una web para que los busca- } \\
\text { dores sean capaces de entenderlo de manera eficaz. ¿La web analizada utiliza } \\
\text { el marcado semántico Schema.org? }\end{array}$ & $0-1$ \\
\hline 4.3 & $\begin{array}{l}\text { Título SEO y me- } \\
\text { tadescripción }\end{array}$ & $\begin{array}{l}\text { El título y la metadescripción son dos etiquetas que sirven para describir el } \\
\text { contenido de la página a los buscadores. El título funciona como un titular } \\
\text { SEO, visible solamente para buscadores, y la metadescripción como un pe- } \\
\text { queño resumen sobre la página ¿La web analizada tiene las etiquetas título y } \\
\text { metadescripción optimizadas? }\end{array}$ & $0-1$ \\
\hline \multicolumn{4}{|c|}{ Parámetro 5: Señales de usuario } \\
\hline ID & Indicador & Descripción & Puntuación \\
\hline 5.1 & $\begin{array}{l}\text { Permanencia en } \\
\text { el sitio }\end{array}$ & $\begin{array}{l}\text { La permanencia en el sitio es el tiempo que un usuario navega por una web. } \\
\text { A mayor tiempo navegando por la web mejor comportamiento se le otorgará } \\
\text { al usuario. ¿Los usuarios que navegan por la web caso de estudio tiene un } \\
\text { comportamiento positivo con un porcentaje alto de permanencia en el sitio? }\end{array}$ & 1 a 8 \\
\hline 5.2 & Tasa de rebote & $\begin{array}{l}\text { La tasa de rebote es el porcentaje de abandono de los usuarios cuando } \\
\text { navegan por una página web. A menor porcentaje de rebote del usuario } \\
\text { mejor comportamiento web. ¿Los usuarios que navegan por la web caso de } \\
\text { estudio tienen un comportamiento positivo con un porcentaje bajo de tasa } \\
\text { de rebote? }\end{array}$ & 1 a 8 \\
\hline 5.3 & Páginas vistas & $\begin{array}{l}\text { Las páginas vistas son el número de páginas por las que navega un usuario } \\
\text { en una web. A mayor número de páginas vistas por el usuario mejor compor- } \\
\text { tamiento web. ¿Los usuarios que navegan por la web caso de estudio tienen } \\
\text { un comportamiento positivo con un porcentaje alto del número de páginas } \\
\text { visitadas? }\end{array}$ & 1 a 8 \\
\hline
\end{tabular}




\begin{tabular}{|c|c|c|c|}
\hline \multicolumn{4}{|c|}{ Parámetro 6: Autoridad } \\
\hline ID & Indicador & Descripción & Puntuación \\
\hline 6.1 & $\begin{array}{l}\text { Número de } \\
\text { backlinks }\end{array}$ & $\begin{array}{l}\text { Los backlinks (enlaces externos) son links que apuntan desde una página web } \\
\text { a otra como fuente de autoridad. ¿La web analizada cuenta con una buena } \\
\text { cantidad de enlaces externos? }\end{array}$ & 1 a 8 \\
\hline 6.2 & $\begin{array}{l}\text { Autoridad de los } \\
\text { backlinks }\end{array}$ & $\begin{array}{l}\text { La autoridad de los backlinks (enlaces externos) proviene del número de } \\
\text { páginas web que enlazan de una web a otra. A mayor autoridad recibida ma- } \\
\text { yores posiciones se obtiene en los motores de búsqueda. ¿La web analizada } \\
\text { recibe enlaces externos de un gran número de páginas web? }\end{array}$ & 1 a 8 \\
\hline 6.3 & $\begin{array}{l}\text { Integración de } \\
\text { redes sociales }\end{array}$ & $\begin{array}{l}\text { La integración de las redes sociales en una web implica incluir los canales } \\
\text { sociales oficiales de la web en forma de enlace para promoverlos y promocio- } \\
\text { narlos para conseguir más seguidores. ¿La web analizada tienen claramente } \\
\text { identificados los iconos/enlaces de sus redes sociales? }\end{array}$ & $0-1$ \\
\hline 6.4 & $\begin{array}{c}\text { Difusión en redes } \\
\text { sociales }\end{array}$ & $\begin{array}{l}\text { Promocionar el contenido de la web en redes sociales es tener un conjunto } \\
\text { de estrategias de dinamización del contenido del sitio web para obtener más } \\
\text { visitas, más lectores, etc. ¿La web analizada publica sus noticias en sus redes } \\
\text { sociales de manera periódica? }\end{array}$ & $0-1$ \\
\hline \multicolumn{4}{|c|}{ Parámetro 7: Buscabilidad } \\
\hline ID & Indicador & Descripción & Puntuación \\
\hline 7.1 & Búsqueda simple & $\begin{array}{l}\text { Se entiende como búsqueda simple al buscador interno de una web en base } \\
\text { a palabras clave para encontrar información ordenada dentro del sitio web. } \\
\text { ¿La web analizada cuenta con un sistema de búsqueda simple por palabras } \\
\text { clave? }\end{array}$ & $0-1$ \\
\hline 7.2 & $\begin{array}{l}\text { Página de } \\
\text { resultados }\end{array}$ & $\begin{array}{l}\text { Se entiende por página de resultados al listado resultante de utilizar el busca- } \\
\text { dor interno de una web. Este listado debe estar ordenado de acuerdo a crite- } \\
\text { rios de relevancia u otros. ¿La web analizada arroja resultados de búsqueda } \\
\text { adecuados y estructurados cuando se utiliza su buscador interno? }\end{array}$ & $0-1$ \\
\hline 7.3 & $\begin{array}{c}\text { Búsqueda interna } \\
\text { avanzada }\end{array}$ & $\begin{array}{l}\text { La búsqueda interna avanzada es una forma de buscar que combina palabras } \\
\text { clave y ecuaciones en forma de criterios de inclusión y exclusión que ayudan } \\
\text { a refinar los resultados de búsqueda. ¿Hay un sistema de búsqueda avanzada } \\
\text { que permita utilizar búsqueda booleana y parametrizada? }\end{array}$ & $0-1$ \\
\hline
\end{tabular}

Fuente: Adaptado de Lopezosa y Codina (2019).

Aunque en la tabla de indicadores hemos aportado una pregunta de chequeo para cada uno de ellos, esta es de tipo conceptual y ayuda por tanto a definir la toma de datos, pero en el momento de la misma, es útil disponer de elementos de decisión pragmáticos adicionales, en especial cuando la puntuación consiste en una escala.

Por ello, a continuación mostramos brevemente el procedimiento de toma de decisiones del PAXBCM para comprobar el cumplimiento relativo de cada uno de los indicadores y poder llegar así a nuestros resultados:

- Navegación estructural: se da por válido cuando la página de portada de la web analizada nos permite acceder a las secciones del sitio en un máximo de tres clics.

- Enlaces semánticos: se cumple este indicador cuando encontramos enlaces en forma de etiquetas o categorías en las noticias del diario nativo digital estudiado.
- Taxonomía: se acepta la existencia de la misma cuando encontramos una estructura de navegación basada en categorías temáticas.

- Enlazado interno: se evalúa positivamente este indicador cuando encontramos en el cuerpo de las noticias enlaces que nos llevan a otras noticias relacionadas con la información que estamos leyendo.

- Extensión: se considera adecuada la extensión cuando las noticias analizadas igualen o superan las 400 palabras.

- Palabras clave: se considera adecuado cuando encontramos en el cuerpo de la noticia una distribución de palabras clave específicas muy relacionadas con el titular y la temática de la información publicada.

- Contenido audiovisual: se puntúa positivamente este indicador cuando se observa contenido audiovisual en las noticias analizadas. 
- Mobile first: para este indicador se utiliza la herramienta Mobile Friendly de Google, adoptando la puntuación que aporta la misma.

- Accesibilidad: para evaluar este indicador nos valemos de la herramienta tawdis.net. Si al examinar la web con esta herramienta aparece un resultado favorable lo damos por adecuado, si no es favorable se marca con cero puntos.

- Página de error 404: para conocer si cuentan con este indicador optimizado usamos una url errónea del sitio web analizado. Si al cargar esta url aparece una página personalizada de error 404, el indicador se considera válido.

- Protocolo robots.txt: se acepta como positivo cuando al incluir la url más la extensión "/ robots.txt" se puede observar este protocolo con una estructura estándar.

- Mapa del sitio xml: se da por válido este indicador cuando al incluir la url más la extensión "/sitemap_index.xml" se puede observar este mapa $\mathrm{xml}$ con una estructura estándar.

- Velocidad de carga: estimamos este indicador utilizando la herramienta Pagespeed de Google, que proporciona tres valores: velocidad lenta, normal y óptima. A la primera, lenta, se otorgan cero puntos; normal, medio punto y óptima, un punto.

- Atributo ALT en la imagen: este indicador se da por válido cuando encontramos la descripción (pie de foto) en las imágenes de las noticias seleccionadas incorporado también en el código fuente.

- Marcado semántico con schema.org: lo identificamos con la extensión del navegador Chrome denominada Structured Data Testing. Si esta herramienta identifica los datos estructurados de forma correcta, consideramos que el indicador es adecuado.

- Título SEO y metadescripción: el procedimiento consiste en realizar una búsqueda en Google de la web a analizar, para ello se utiliza como palabra clave el nombre del diario nativo digital. El indicador se acepta si los resultados de la primera página corresponden a dicha web y cuentan con títulos y descripciones coherentes correspondientes a la etiqueta $<$ title $>$ y al metadato <description $>$.

- Permanencia en el sitio, tasa de rebote y páginas vistas: estos resultados se obtienen utilizando la herramienta Alexa de Amazon.
Los mejores resultados de todos los diarios nativos digitales analizados obtienen las mejores puntuaciones y viceversa.

- Número de backlinks y autoridad de los backlinks: estos datos se obtienen utilizando la herramienta SEMrush. A mayor número de enlaces recibidos y a mayor cantidad de webs que enlazan a los diarios nativos digitales analizados mayor puntuación.

- Integración de redes sociales y difusión en redes sociales: para considerar adecuado este indicador las webs analizadas deben tener sus propias redes sociales y difundir su contenido en ellas.

- Búsqueda simple, página de resultados y búsqueda avanzada: para evaluar estos indicadores se realiza una consulta simple (un concepto) y avanzada (varios conceptos) del buscador interno de las webs analizadas. Estos indicadores se consideran adecuados si el sistema acepta las búsquedas y se presentan resultados adecuados a dichas consultas.

Una vez descrita la metodología empleada, los parámetros que componen el protocolo PAXBCM y el procedimiento a seguir para puntuar el grado de optimización del SXO de cada diario nativo digital analizado, pasamos a presentar los resultados obtenidos.

\section{RESULTADOS Y DISCUSIÓN}

En este apartado, en primer lugar, se muestran los resultados de los diferentes diarios nativos digitales (Tabla IV, Figura 1) después de aplicar los indicadores SXO. En segundo lugar, se incluyen las medidas de impacto de la optimización de la experiencia de los usuarios en cada cibermedio. Finalmente, en base a estos resultados, se proponen una serie de recomendaciones para la mejora de la SXO.

Como podemos ver, el diario nativo digital que presenta una mejor experiencia de búsqueda de modo global es libertaddigital.com con 50 puntos en total, debido, sobre todo, al buen rendimiento en el parámetro 5-Señales de usuario, y unos buenos resultados generales en los demás parámetros.

Le siguen elconfidencial.com con 47,5 puntos, publico.es con 46 puntos, diario.es con 44 puntos, elespanol.com con 40 puntos, vozpopuli.com con 34 puntos, huffingtonpost.es, e infolibre.es con 24 puntos.

A partir de los resultados agregados, vamos a revisar ahora la situación de cada uno de los parámetros. 
Tabla IV. Resultados agregados

\begin{tabular}{|c|c|c|c|c|c|c|c|c|c|}
\hline \multicolumn{10}{|c|}{ Parámetro 1: Arquitectura de la información } \\
\hline No & Indicador & M1 & M2 & M3 & M4 & M5 & M6 & M7 & M8 \\
\hline 1.1 & Navegación estructural & 1 & 1 & 1 & 1 & 1 & 1 & 1 & 1 \\
\hline 1.2 & Enlaces semánticos & 1 & 1 & 1 & 1 & 1 & 1 & 0 & 1 \\
\hline 1.3 & Taxonomía & 1 & 1 & 1 & 1 & 1 & 1 & 1 & 1 \\
\hline 1.4 & Enlazado interno & 1 & 0 & 1 & 1 & 1 & 1 & 0 & 0 \\
\hline \multicolumn{10}{|c|}{ Parámetro 2: SEO de contenidos } \\
\hline No & Indicador & M1 & M2 & M3 & M4 & M5 & M6 & M7 & M8 \\
\hline 2.1 & Extensión & 1 & 1 & 1 & 1 & 1 & 1 & 1 & 1 \\
\hline 2.2 & Palabras clave & 1 & 1 & 1 & 1 & 1 & 1 & 1 & 1 \\
\hline 2.3 & Contenido audiovisual & 0 & 0 & 0 & 0 & 1 & 0 & 0 & 0 \\
\hline 2.4 & Mobile First & 1 & 1 & 1 & 1 & 1 & 1 & 1 & 1 \\
\hline 2.5 & Accesibilidad & 0 & 0 & 0 & 0 & 0 & 0 & 0 & 0 \\
\hline \multicolumn{10}{|c|}{ Parámetro 3: SEO técnico } \\
\hline No & Indicador & M1 & M2 & M3 & M4 & M5 & M6 & M7 & M8 \\
\hline 3.1 & Página de error 404 & 1 & 1 & 1 & 1 & 1 & 1 & 1 & 1 \\
\hline 3.2 & Protocolo robots.txt & 1 & 1 & 1 & 1 & 1 & 1 & 1 & 1 \\
\hline 3.3 & Mapa del sitio xml & 1 & 1 & 0 & 1 & 1 & 1 & 0 & 1 \\
\hline 3.4 & Velocidad de carga & 0 & 0 & 0.5 & 0.5 & 0 & 0 & 0 & 0 \\
\hline \multicolumn{10}{|c|}{ Parámetro 4: Metadatos } \\
\hline No & Indicador & M1 & M2 & M3 & M4 & M5 & M6 & M7 & M8 \\
\hline 4.1 & Atributo ALT en la imagen & 1 & 1 & 1 & 1 & 1 & 1 & 1 & 1 \\
\hline 4.2 & Marcado semántico con schema.org & 1 & 1 & 1 & 1 & 1 & 1 & 1 & 1 \\
\hline 4.3 & Título SEO y metadescripción & 1 & 1 & 1 & 1 & 1 & 1 & 1 & 1 \\
\hline \multicolumn{10}{|c|}{ Parámetro 5: Señales de usuario } \\
\hline No & Indicador & M1 & M2 & M3 & M4 & M5 & M6 & M7 & M8 \\
\hline 5.1 & Permanencia en el sitio & 4 & 7 & 6 & 3 & 2 & 1 & 8 & 5 \\
\hline 5.2 & Tasa de rebote & 4 & 7 & 5 & 3 & 1 & 2 & 8 & 6 \\
\hline 5.3 & Páginas vistas & 5 & 6 & 7 & 4 & 2 & 1 & 8 & 3 \\
\hline \multicolumn{10}{|c|}{ Parámetro 6: Autoridad } \\
\hline No & Indicador & M1 & M2 & M3 & M4 & M5 & M6 & M7 & M8 \\
\hline 6.1 & Número de backlinks & 6 & 4 & 5 & 8 & 3 & 1 & 7 & 2 \\
\hline 6.2 & Autoridad de los backlinks & 7 & 6 & 8 & 5 & 3 & 1 & 4 & 2 \\
\hline 6.3 & Integración de redes sociales & 1 & 1 & 1 & 1 & 1 & 1 & 1 & 1 \\
\hline 6.4 & Difusión en redes sociales & 1 & 1 & 1 & 1 & 1 & 1 & 1 & 1 \\
\hline \multicolumn{10}{|c|}{ Parámetro 7: Buscabilidad } \\
\hline No & Indicador & M1 & M2 & M3 & M4 & M5 & M6 & M7 & M8 \\
\hline 7.1 & Búsqueda simple & 1 & 1 & 1 & 1 & 1 & 1 & 1 & 1 \\
\hline 7.2 & Página de resultados & 1 & 1 & 1 & 1 & 1 & 1 & 1 & 1 \\
\hline 7.3 & Búsqueda interna avanzada & 1 & 0 & 0 & 0 & 1 & 1 & 1 & 0 \\
\hline & Puntuación total & 44 & 46 & 47,5 & 40 & 30 & 24 & 50 & 34 \\
\hline
\end{tabular}

M1: diario.es, M2: publico.es, M3: elconfidencial.com, M4: elespanol.com, M5: Huffingtonpost.es, M6: Infolibre.es, M7: libertaddigital.com, M8: Vozpopuli.com. 
Figura 1. Puntuación resultante del estudio de análisis de la experiencia de búsqueda de los 8 diarios nativos digitales analizados utilizando el protocolo PAXBCM

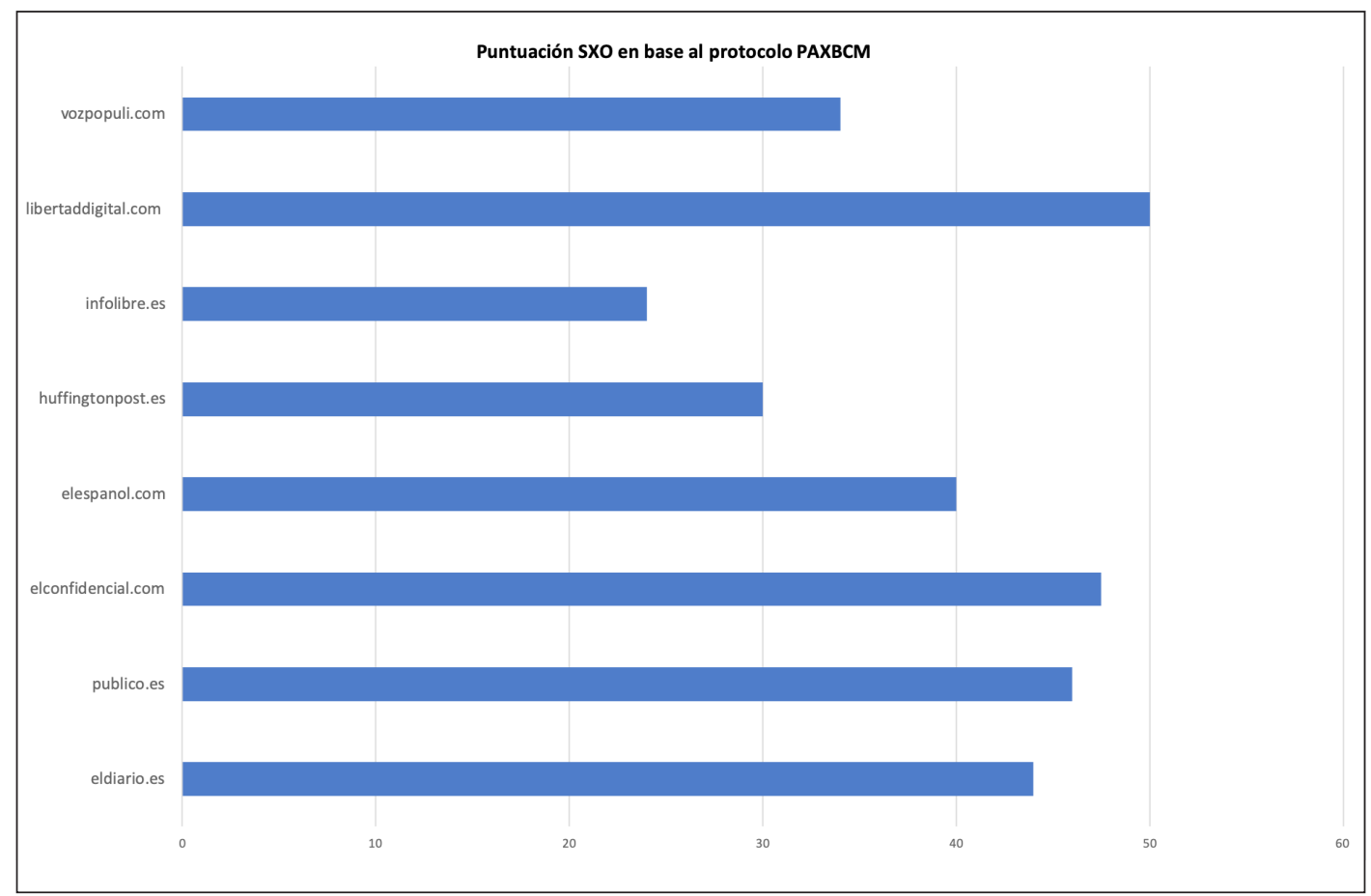

\section{Parámetro 1: Arquitectura de la información}

Diario.es, elconfidencial.com, elespanol.com, huffingtonpost.es, e infolibre.es son los diarios nativos digitales que cuentan con una arquitectura de la información optimizada tanto para su versión móvil como para su versión para ordenador. Sin embargo, libertaddigital.com, publico.es y vozpopuli.com deberían favorecer el uso de enlaces ya que de este modo ayudaría a los usuarios que podrían completar el contenido que leen favoreciendo así su experiencia de lectura.

\section{Parámetro 2: SEO de contenidos}

Si bien es cierto que todos los nativos digitales analizados cumplen en mayor o menor medida con este parámetro, también es cierto que todos fallan en el campo de la accesibilidad. Esto puede tener varias lecturas, por un lado, que se considere de gran complejidad la adaptación del contenido de un cibermedio a usuarios discapacitados, en particular a invidentes, y por otro lado, que no se contemple este tipo de usuario como publico objetivo (target) al que va a dirigir sus informaciones. Aun con estas lecturas, y teniendo en cuenta ya no solo el SXO sino la labor social del periodismo, este indicador debería aplicarse y cumplirse mucho mejor dentro del sector. Por otro lado, siete de los ocho diarios nativos digitales analizados no contemplan la producción de noticias de texto con vídeos. Todos incluyen contenido audiovisual, pero algunos de modo muy casual y por tanto no se puede afirmar que forme parte de sus rutinas productivas. Solo Huffingtonpost. es, en cambio, está apostando por el contenido audiovisual, con ello no solo está enriqueciendo el contenido de sus noticias, sino que están ofreciendo distintas alternativas para que los usuarios se mantengan informados.

\section{Parámetro 3: SEO técnico}

Los resultados demuestran que todos los diarios nativos digitales analizados consideran importante la aplicación de acciones de SEO técnico sobre todo en los aspectos relacionados con los motores de búsqueda, aunque no tanto con los que tienen que ver con el usuario. Esto se ve claramente en que todos los cibermedios analizados cuentan con un fichero robots.txt y casi todos tienen un sitemap para facilitar el trabajo de rastreo e indexación a los buscadores. Sin embargo, ninguno de los cibermedios analizados cuentan con una velocidad de carga optimizada tanto para su versión para ordenador como para 
su versión móvil, lo cual va en detrimento de la experiencia del usuario que debe esperar a que se cargue la página a visitar por encima de la media de tiempo internacional y de otras clases de sitios web.

\section{Parámetro 4: Metadatos}

Todos los diarios nativos digitales obtienen la máxima puntuación en este parámetro, aplicando favorablemente todos los indicadores analizados. Esto demuestra el auge y la importancia de los metadatos en cibermedios como estrategia para posicionar sus noticias en los motores de búsqueda, También se confirma una estandarización de la tecnología de marcado semántico schema.org para hacer que el contenido sea más inteligible para los buscadores. Los principales datos estructurados de schema.org utilizados por los diarios nativos digitales analizados son:

- NewsArticle: datos estructurados que identifican semánticamente que el contenido de las webs analizadas corresponde a noticias.

- Webpage y Website: datos estructurados que hacen entender a los motores de búsqueda que los diarios nativos digitales analizados son páginas web.

- Organization: datos estructurados ajustados a la descripción empresarial de los medios de comunicación analizados, ayudando así a los motores de búsqueda a alinear un diario nativo digital con su correspondiente grupo empresarial de comunicación.

- ImageGallery e Imageobject: datos estructurados que definen las imágenes y galería de imágenes que se incluyen en las noticias.

\section{Parámetro 5: Señales de usuario}

En líneas generales se muestra un tiempo de permanencia alta, una baja tasa de rebote y una buena navegación, fiel reflejo sin duda del enorme impacto de estos indicadores en el SEO. Libertaddigital.com es el diario nativo digital que cuenta con una mejor interacción de sus usuarios en cuanto a tiempo de navegación, número de páginas vistas (páginas visitadas por cada usuario) y tasa de rebote (porcentaje de abandono de visitantes cuando entran en una página web), ya que permanecen en su sitio web una media de 6:20 minutos, tiene una tasa de rebote del $46.30 \%$ y sus usuarios visitan un promedio de 2.63 páginas.

El ranking de estos tres indicadores se muestra a continuación:
- Los usuarios de libertaddigital.com permanecen en su sitio web una media de 6:20 minutos, le siguen los usuarios de publico. es con 5:17 minutos, elconfidencial.com con 4:58 minutos, vozpopuli.com con $4: 15 \mathrm{mi}-$ nutos, diario.es con 4:11 minutos, elespanol.com con 2:50 minutos, huffingtonpost. es con 2:49 minutos, e infolibre.es con 2:47 minutos.

- Libertaddigital.com, como ya hemos señalado, tiene la mejor tasa de rebote con un $46.30 \%$, le siguen los usuarios de Publico. es con el $55.60 \%$, vozpopuli.com con un $60.60 \%$, elconfidencial.com con el $61.10 \%$, diario.es con un $63.70 \%$, elespanol.com con un $72 \%$, infolibre.es con un $74.90 \%$ y huffingtonpost.es con un $75 \%$.

- Los usuarios de libertaddigital.com visitan una media de 2.63 páginas de este medio, le siguen los usuarios de elconfidencial.com con 2.20 páginas, publico.es con 2.12 páginas, diario.es con 1.97 páginas, elespanol. com con 1.93 páginas, vozpopuli.con con 1.80 páginas, huffingtonpost.es con 1.72 páginas, e infolibre.es con 1.44 páginas.

Como constatan estos datos existen grandes diferencias entre el diario con mejor puntuación y el de peor puntuación. En este sentido infolibre. es (el que ha obtenido la puntuación más baja en este parámetro) debería considerar la calidad de su contenido, el tipo de lectores que visitan su web, y sobre todo reorganizar sus enlaces y su navegación para favorecer que los lectores pasen más tiempo en su web, visitando más páginas internas.

\section{Parámetro 6: Autoridad}

Este parámetro demuestra que la prensa digital es una fuente de referencia importante por los muchos otros sitios web que les enlazan, ya que encontramos con millones de enlaces de cientos de webs que transfieren autoridad a estos cibermedios. De hecho, elespanol.com y elconfidencial. com son los dos diarios nativos digitales con mayor autoridad web, por un lado, elespanol.com es la web que recibe mayor número de enlaces externos y por otro lado, elconfidencial.com es el diario nativo digital con mayor número de webs externas que le enlazan.

El ranking de estos dos indicadores se muestra a continuación:

- Elespanol.com es el portal web que recibe mayor número de enlaces externos, con un total de 34,2 millones, le siguen libertaddigital.com con 29,3 millones, diario.es con 17,7 millones, 
elconfidencial.com con 16.5 millones, publico. es con 13,4 millones, huffingtonpost.es con 7 millones, vozpopuli.com con 2,6 millones e infolibre.es con 868.752 enlaces.

- Elconfidencial.com es el diario nativo digital que es enlazado por mayor número de webs externas, concretamente 39.597 sitios webs, seguido de diario.es con 33.881, publico.es con 31.914, elespanol.com con 22.514, libertaddigital.com con 17.409, huffingtonpost.es con 15.505, vozpopuli.com con 8.883 e infolibre.es con 4.723 .

Por otra parte, todos los diarios nativos digitales analizados cuentan con canales sociales y difunden su contenido a través de ellos. De nuestro análisis se confirma que todos apuestan por Facebook y Twitter pero también se ven nuevas tendencias apuestas hacia otras redes sociales como Instagram, Youtube, Google+, Telegram, LinkedIN y Flipboard.

\section{Parámetro 7: Buscabilidad}

Por último, los buscadores internos funcionan perfectamente en todos los diarios nativos digitales analizados cuando se realiza una consulta básica. Pero solo la mitad, concretamente, libertaddigital.com, diario.es, huffingtonpost.es e infolibre.es, poseen una implementación óptima de las búsquedas avanzadas. No tener un buscador optimizado para búsquedas avanzadas o tenerlo, pero que dé resultados erróneos, denota que el papel de se les da a estos buscadores internos es muy secundario. Ahora bien, conformarse con ofrecer resultados de noticias internas con búsquedas simples supone una oportunidad perdida para estos cibermedios ya que no pueden conocer y testear temas complejos que puedan interesar a ciertos usuarios, y tampoco pueden ayudar a una mejor interacción entre el que busca y las noticias que encuentra.

\section{CONCLUSIONES}

A continuación, examinaremos los objetivos y las preguntas de investigación y presentaremos las conclusiones, así como al final presentaremos también sugerencias para nuevas investigaciones.

En primer lugar, es importante destacar que una alta o baja puntuación del ranking de experiencia de búsqueda no tiene relación directa con una buena 0 mala calidad de la información que publican estos medios. Port tanto, cabe reforzar que, lo que miden estos resultados es, estrictamente, un buen desempeño en el SXO. Otra cuestión es que resulte vital para el encaje del medio en el ecosistema digital.
En concreto, los resultados indican buen SXO general, lo cual no implica necesariamente buen periodismo. Este es un motivo, precisamente, para reclamar del mejor periodismo que no descuide el SXO. Los periodistas y los ciudadanos tienen derecho a esperar que el mejor periodismo se preocupe tanto por la visibilidad, como por la buscabilidad, según argumentos que ya hemos presentado más arriba (Cutts, 2012; Potter, 2012; Villanueva, 2013; Munroe, 2015; Arora, 2016; Chasinov, 2017).

En cualquier caso, para presentar nuestras conclusiones, vamos a retomar los objetivos de este trabajo, que eran los siguientes:

Objetivo 1. Identificar los componentes del $\mathrm{SXO}$, tanto en la versión móvil como ordenador de escritorio, en los principales diarios nativos digitales de España y determinar la eficacia de su aplicación:

Hemos podido identificar los principales componentes del SXO y además hemos demostrado que es factible llevar a cabo un sistema de análisis del SXO tomando los parámetros que componen el protocolo PAXBCM. Estos se enfocan en el acceso a la información interna de la web, el buen o mal uso del SEO de contenidos, la buena o mala optimización del SEO técnico, la optimización de los metadatos, la identificación de las principales métricas de señales de usuario (estancia en el sitio, páginas vistas y tasa de rebote), la autoridad web en base al número de enlaces externos obtenidos y el número de páginas webs externas que les enlazan y la eficaz o ineficaz buscabilidad interna tanto para consultas simples como avanzadas.

Gracias a estos parámetros hemos llevado a cabo un análisis comparativo en ocho diarios nativos digitales, con resultados muy diferentes para cada medio, pero que, a su vez, nos ha permitido reconocer que cuentan en general con una estrategia de optimización de la experiencia del usuario que ayuda al posicionamiento y la visibilidad web en motores de búsqueda. De hecho, hemos podido constatar que todos los diarios nativos digitales analizados llevan a cabo acciones de SXO en un porcentaje bastante elevado, con buenos resultados en los siete parámetros que se incluyen dentro del protocolo utilizado para este análisis.

Objetivo 2. Desarrollar y llevar a cabo un análisis comparativo con capacidad para proporcionar un ranking que identifique los medios que llevan a cabo mejores prácticas en los diferentes parámetros del SXO.

Algunos de los mejores resultados obtenidos se han centrado en la arquitectura de la información, el SEO de contenidos, el SEO semántico, las seña- 
les de los usuarios y la autoridad web. De todos ellos hemos podido confeccionar un ranking de mayor a menor grado de aplicación del SXO lo que nos ha proporcionado un mapeo del desempeño de los cibermedios analizados.

En este sentido, el diario nativo digital que presenta una mejor experiencia de búsqueda global y que, por tanto, ocupa la primera posición del ranking es libertaddigital.com con 50 puntos, seguidos delconfidencial.com con 47,5 puntos, publico.es con 46 puntos, diario.es con 44 puntos, elespanol. com con 40 puntos, vozpopuli.com con 34 puntos, huffingtonpost.es, e infolibre.es con 24 puntos.

Objetivo 3. Proponer recomendaciones de SXO susceptibles de aplicarse a medios de comunicación digitales.

Nuestro estudio de caso ha permitido identificar estrategias y patrones de optimización de la experiencia de usuarios en diarios nativos digitales que, además, pueden aplicarse a otros cibermedios.

En concreto, tras el análisis de los ocho diarios nativos digitales seleccionados y de sus resultados, hemos podido identificar una serie de recomendaciones para la optimización del SXO. En este sentido, algunas de las mejores estrategias SXO que se pueden aplicar dentro del sector cibermedios son la adecuación de las estructuras de navegación, la creación de contenido que incluya componentes multimedia y el uso palabras clave derivadas que enriquezcan las noticias. También la utilización del marcado semántico Schema.org, la adecuada optimización de las etiquetas ALT, el uso de título y metadescripción, la obtención de enlaces externos de calidad, la mejora del tiempo de estancia de los usuarios en la web y el número de páginas internas por las que navega, para reducir así la tasa de rebote. Por último, estos cibermedios deben contar con un buscador interno que funcione adecuadamente cuando los usuarios realicen tanto búsquedas simples como búsquedas complejas, apartado este último en que falla la mitad de ellos.

Por su parte, la pregunta de investigación de este trabajo, con la revisión de la cual queremos completar las conclusiones, era la siguiente:

¿Cuáles son los componentes más significativos del SXO, cuál es el grado de observación e implementación de estos componentes que presentan los diarios nativos digitales españoles y cuál es la posición relativa de cumplimiento entre ellos?

Como respuesta, podemos señalar que los $8 \mathrm{ci}$ bermedios analizados tienen margen de mejora variable según los casos en cuanto a su SXO, sobre todo en los patrones de SEO de contenidos, SEO técnico y buscabilidad, pero sin dejar de lado los demás parámetros (arquitectura de la información, autoridad, señales sociales y metadatos).

Así mismo, del análisis realizado podemos confirmar que los indicadores más débiles son la velocidad de carga de la web, y su adaptación a las normas de accesibilidad para permitir que al sitio puedan acceder todo tipo de usuarios. También deben mejorar en el uso de contenido multimedia/ audiovisual en las noticias y deberían contar con un buscador interno que funcione adecuadamente para búsqueda avanzadas.

En estos dos aspectos debemos insistir que los cibermedios analizados (en medida variable) tienen amplios márgenes de mejora. En el caso de la accesibilidad, la función social de los medios de comunicación debería asegurar una mayor sensibilidad para incorporar a todos los usuarios, incluyendo los que poseen alguna discapacidad.

Como resumen, el ranking lo lidera libertaddigital.com con 50 puntos sobre 61 , seguido de elconfidencial.com con 47,5 puntos y publico.es con 46 puntos. Por el contrario, infolibre.es es el diario nativo digital con una menor integración del SXO ocupando el último puesto del ranking con 24 puntos.

En definitiva, se confirma que el protocolo de análisis denominado PAXBCM y del que hemos mostrado los indicadores que permiten llevar a cabo la toma de datos permite conducir análisis del SXO en cibermedios.

Por tanto, creemos que puede ser de utilidad tanto a responsables del SEO tradicional de medios de comunicación que necesiten instrumentos de auditoría interna, como a estudiosos de los cibermedios que necesiten llevar a cabo análisis comparativos de cualquier tipo.

Por la misma razón, creemos que es posible diseñar futuras investigaciones que extiendan la aplicación de este sistema de análisis, o alguna variación del mismo, a otros sectores de la comunicación.

Por ejemplo, se podría realizar un estudio comparativo del SXO entre diarios nativos digitales y diarios digitales con tradición en la prensa de papel para conocer las similitudes y diferencias en los elementos analizados entre ambos tipos de diarios digitales. En definitiva, se trataría, por tanto, de una posible línea de estudios con una indudable utilidad social puesto que, además de ayudar a caracterizar de manera más eficaz determinados sectores de la comunicación digital, ayuda a su optimización continuada para una mejora para los ciudadanos. 


\section{AGRADECIMIENTOS}

Este trabajo forma parte del proyecto «Creación y contenido interactivo en la comunicación de información audiovisual: audiencias, diseño, sistemas y formatos» (CSO2015-64955-C4-2-R), subvencionado por el Ministerio de Economía y Competitividad, (MINECO/Feder), España, Plan Nacional de I+D+i.

\section{REFERENCIAS}

Arora, L. (2016). SEO: Search Experience Optimization. Disponible en: https://www.envigo.co.uk/blog/searchengine-optimisation/seo-search-experience-optimization [Fecha de consulta: 09/11/2018].

Asser, M. (2012). Search Engine Optimisation in BBC News. Disponible en: http://www.bbc.co.uk/blogs/internet/posts/search_engine_optimisation_in

Charlton, G. (2016). How much do journalists and editors need to know about SEO?. Disponible en: https:// searchenginewatch.com/2016/03/15/how-much-dojournalists-and-editors-need-to-know-about-seo/

Chasinov, N. (2017). Search Experience Optimization: Welcome to the New SEO. Disponible en: https:// www.huffingtonpost.com/young-entrepreneurcouncil/search-experience-optimiz_b_10378498. html?guccounter=1 [Fecha de consulta: 09/11/2018].

Codina, L. (2000). Evaluación de recursos digitales en línea: conceptos, indicadores y métodos. Revista Española de Documentación Científica, 23 (1), 9-44. https://doi.org/10.3989/redc.2000.v23.i1.315

Codina, L.; Pedraza, R.; Díaz-Noci, J.; Rodríguez-Martínez, R.; Pérez-Montoro, M.; Cavaller-Reyes, V. (2014). Sistema Articulado de Análisis de Cibermedios (SAAC): Una propuesta sobre el qué y el cómo para estudiar medios de comunicación digitales. Hipertext.net, 12, e018. https://doi.org/10.2436/20.8050.01.13

Codina, L. (2018). Componentes Estructurales y Semánticos en el Diseño de la Navegación Web. Taxonomías, SEO y Software implicado en el caso de una Instalación con WordPress. Disponible en: https://repositori.upf. edu/bitstream/handle/10230/27879/codina_2017_ comp.pdf?sequence $=1$ \&isAllowed $=y$ [Fecha de consulta: $07 / 11 / 2018]$.

Coller, X. (2000). Estudio de Casos, Colección Cuadernos Metodológicos. Madrid: Editorial Centro de Investigaciones Sociológicas.

Cutts, M. (2012). Do you think that Search Engine Optimization should be renamed? [video en canal de YouTube] Disponible en: https://www.youtube. com/watch?v=ZStQhWx8YPc [Fecha de consulta: 09/11/2018].

Eisenhardt, K. (1989). Building theories from case study research. Academy of Management Review, 14 (4), 532-550. https://doi.org/10.5465/ amr.1989.4308385
Work is a part of the project «Creación y contenido interactivo en la comunicación de información audiovisual: audiencias, diseño, sistemas y formatos» (CSO2015-64955-C4-2-R), subsidized by Ministerio de Economía y Competitividad, (MINECO/ Feder), Spain, Plan Nacional de I+D+i.

Gandhi, M. (2017). Why and How to Deliver the Best Search Experience in 2018. Disponible en: https:// www.seoclarity.net/blog/search-experience-optimization-2018-17568/ [Fecha de consulta: 10/11/2018]

García-Carretero, L.; Codina, L.; Díaz-Noci, J.; IglesiasGarcía, M. (2016). Herramientas e indicadores SEO: características y aplicación para análisis de cibermedios. El Profesional de la Información, 25 (3), 497-504. http://dx.doi.org/10.3145/epi.2016.may.19

Giomelakis, D.; Veglis, A. (2015a). Employing Search Engine Optimization techniques in Online News. Studies in Media and Communication, 3 (1). http://dx.doi. org/10.11114/smc.v3i1.683

Giomelakis, D.; Veglis, A. (2015b). Investigating Search Engine Optimization Factors in Media Websites, The case of Greece. Digital Journalism, 4 (3), pp. 379-400. https://doi.org/10.1080/21670811.2015.1046992

Guallar, J.; Abadal, E. Codina, L. (2012). Sistema de análisis de hemerotecas de prensa digital. Trípodos, 31, 37-64.

Hassan, Y.; Martín-Fernández, F.; Iazza, G. (2004). Diseño Web Centrado en el Usuario: Usabilidad y Arquitectura de la Información. Disponible en: https://www. upf.edu/hipertextnet/numero-2/diseno_web.html [Fecha de consulta: 10/11/2018].

Iglesias-García, M. y Codina, L. (2016). Los cibermedios y la importancia estratégica del posicionamiento en buscadores (SEO). Opción, 32, 929-944. Disponible en: https://www.redalyc.org/pdf/310/31048482052.pdf

Jiménez, L.; Aguilar, C.; Sánchez, L.; Pérez-Montoro, M. (2018). User experience and the media: The threeclick rule on newspaper websites for smartphones. Revista Latina de Comunicación Social, 73, 595-613. https://doi.org/10.4185/RLCS-2018-1271en

Kukoo, I. (2016). SEO Should Stand For Search Experience Optimization. Disponible en: https://dee.ie/it-blog/ seo-should-stand-for-search-experience-optimization/ [Fecha de consulta: 11/11/2018].

Lemos, J.; Yoseph, J. (2017). Search Engine Optimization to Enhance User Interaction. 2017 International Conference on I-SMAC (IoT in Social, Mobile, Analytics and Cloud) (ISMAC). Palladam, India: IEEE. https://doi.org/10.1109/ I-SMAC. 2017.8058379.

Linares, J.; Codina, L.; Abadal, E.; Guallar, J. (2016). Periodismo en bases de datos y buscabilidad de la información: Protocolo de análisis, y resultados de 
aplicación. Barcelona: Departamento de Comunicación (UPF), Serie Editorial DigiDoc. Acceso: http://hdl. handle.net/10230/27310

Liu, X. (2017). Large-scale SEO Optimization Algorithm based on Transfer Learning. Boletín Técnico, 55 (11), 587-593.

Lopezosa, C.; Codina, L.; Caldera-Serrano, J. (2018). SEO semántico: Framework ISS para la optimización de sitios intensivos en contenidos. Cuadernos de Documentación Multimedia, 29, 97-122. https://doi. org/10.5209/CDMU.60607

Lopezosa, C. ; Codina, L. (2020). Protocolo de Análisis de la eXperiencia de Búsqueda en CiberMedios (PAXBCM). En Pedraza, R.; Codina, L.; Guallar, J. (coords.), Calidad en sitios web. Barcelona: Ed. UOC.

Manish, M.; Roohi, A. (2013). Evolution of Search Engine Optimization and Investigating the Effect of Panda Update into it. International Journal of Scientific \& Engineering Research, 4 (12), 2013- 2045.

Morato, J. ; Sánchez-Cuadrado, S.; Moreno, V.; Moreiro, J. A. (2013). Evolución de los factores de posicionamiento web y adaptación de las herramientas de optimización. Revista Española de Documentación Científica, 36 (3), e018. http://dx.doi.org/10.3989/redc.2013.3.956

Munroe, M. (2015). From SEO To SXO: Search Experience Optimization. Disponible en: https://searchengineland.com/seo-sxo-search-experience-optimization-223812 [Fecha de consulta: 12/11/2018].

Nuño, M (2014). Sistemas de acceso y consulta en los diarios digitales españoles. Investigación bibliotecológica, 28, 81-99. https://doi.org/10.1016/S0187$358 \times(14) 72567-5$

Ok Diario (2018). OKDIARIO es el séptimo diario más leído de España con 50.1 millones de visitas mensuales. Disponible en: https://okdiario.com/audiencia/comscorejunio-2018-2683229 [Fecha de consulta: 26/01/2019].

Ok Diario (2016). Consulta aquí el top 50 del ranking de comScore multiplataforma del mes de diciembre. Disponible en: https://okdiario.com/audiencia/consulta-aqui-top-50-del-ranking-comscore-multiplataforma-del-mes-diciembre-62504 [Fecha de consulta: 26/01/2019].
Park, M. (2018). SEO for an open access scholarly information system to improve user experience. Information Discovery and Delivery, 46 (2), 77-82. https:// doi.org/10.1108/IDD-08-2017-0060

Pérez-Montoro, M.; Codina, L. (2016). Navigation Design and SEO for Content-Intensive Websites: A Guide for an Navigation Design and SEO for an efficient digital communication. Cambridge: Chandos Publishing. https://doi.org/10.1016/B978-0-08-100676-4.00006-7

Potter, B. (2012). SEO: search experience optimisation. Disponible en: https://econsultancy.com/seo-search-experience-optimisation [Fecha de consulta: 13/11/2018].

Prensa PR (2018). Elpais.com aprovecha septiembre para adelantar a Elmundo.es. Disponible en: https://prnoticias.com/prensa/prensa-pr/20170319-periodicos-digitales-mas-leidos-septiembre-2018-comscore [Fecha de consulta: 11/11/2018].

Sampedro, V. (2018). Dietética digital para adelgazar al gran hermano. Barcelona: Icaria Editorial.

Smyrnaios, N. (2015). Google and the Algorithmic Infomediation of News. Media Fields Journal, no. 10, 1-10.

Templeman, M. (2016). SEO Has Evolved To Search 'Experience' Optimization. Disponible en: https://www.forbes. com/sites/miketempleman/2016/02/16/seo-has-evolved-to-search-experience-optimization/\#5b7735af3f35 [Fecha de consulta: 11/11/2018].

Themistoklis, M.; Symeonidis, A. (2015). Identifying valid search engine ranking factors in a Web 2.0 and Web 3.0 context for building efficient SEO mechanisms. Engineering Applications of Artificial Intelligence, 41, 7591. https://doi.org/10.1016/j.engappai.2015.02.002

Van Dyke, P. (2012). Search Engine Optimization VS. User Experience Optimization. Disponible en: https://www. seo.com/blog/search-engine-optimization-vs-user-experience-optimization/ [Fecha de consulta: 13/11/2018].

Villanueva, L. (2013). Search Experience Optimization. Otra forma de entender el SEO. Disponible en: https:// luismvillanueva.com/seo/search-experience-optimization-seo-sxo.html [Fecha de consulta: 14/11/2018].

Yin, R. (2014). Case Study Research. Design and Methods. Canadá: SAGE. 